screening assessment is not entirely accurate, road testing patients is recommended.

The present system allows a substantial proportion of unsafe drivers to resume driving. This could be reduced by requiring a short, simple cognitive assessment.
Bull JP, Raffle PAB. The ageing driver. Y Royal Soc Med 1992;85:188-9. 2 Nouri FM, Tinson DJ, Lincoln NB. Cognitive ability and driving after stroke. Intomational Disability Studies 1987;9:110-5.

3 Nouri FM, Lincoln NB. Validation of a cognitive asseasment predicting driving performance after stroke. Clinical Rehabilitation 1992;6:275-81.

4 Driving and Pastinson's disease (editorial). Lancot 1990;336:781.

(Accepted 3 Yune 1993)

\section{Remission of seizures in untreated epilepsy}

\section{T Keränen, P J Riekkinen}

Department of Neurolosy, Univeraity Hospital of Kuopio, SF-70210 Kuopio, Finland

T Kerinen, consultant neurologist

PJ Riekkinen, professor

Correspondence to: $\operatorname{Dr} T$ Kerthen, University Hospital of Tampere, SF-33520 Tampere, Finland

BME 1993;307:483

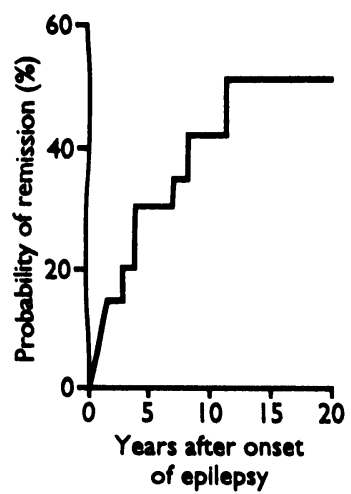

Probability of remission in 33 people with untreated epilepsy
Little is known about the prognosis of untreated epilepsy because medical treatment for seizures has been available for more than a century. Gowers concluded that spontaneous remission in epilepsy would be very rare, ${ }^{1}$ but Zielinski found that $30 \%$ of people with untreated epilepsy became seizure free.

The present study is part of an epidemiological survey in eastern Finland. ${ }^{3}$ We retrospectively analysed seizure outcome in epileptic patients who had never used any antiepileptic drug treatment.

\section{Patients, methods, and results}

All patients with at least two unprovoked non-febrile seizures born before 1 January 1964 and residing in the Kuopio University Hospital district were identified. We reviewed hospital and outpatient records and had access to the files of the Social Insurance Institution as well as contacts with community health centres. A total of 1375 epileptics were identified. Of these, 1220 patients were examined by TK.

The study included people with epilepsy who had never been treated with antiepileptic drugs, had an onset of epilepsy between 1960 and 1977, and had at least two years' follow up at the date of analysis (31 December 1979). Terminal remission was defined as a seizure free period of at least two years extending until the date of analysis. The probability of remission was evaluated by the life table method.

Of the patients examined, $50(4 \cdot 1 \%)$ had never used any antiepileptic drugs. Of these, 33 fulfilled the criteria applied in the present study. The remaining 17 patients were excluded from the analysis because the data available on seizure prognosis were inappropriate. Twenty five of the patients $(76 \%)$ were men. The aetiology of epilepsy was unknown in 25 cases $(76 \%)$ and an organic cause was diagnosed in eight cases $(24 \%)$. Tonic-clonic seizures were diagnosed in 13 patients $(39 \%)$. Eight patients $(24 \%)$ had complex partial seizures, seven patients $(21 \%)$ has partial secondarily generalised seizures, and one patient (3\%) had simple partial seizures. In four patients $(12 \%)$, seizures remained unclassifiable.

The figure shows the probability of terminal remission of seizures in the 33 untreated epileptic patients. The probability of remission was $42 \%$ at 10 years and $52 \%$ at 20 years after the onset of epilepsy.

\section{Comment}

Our results suggest that untreated epilepsy may run a benign course in some patients. The likelihood of subsequent seizures in our untreated epileptic patients was low, in agreement with the findings of Zielinski. ${ }^{2}$ We cannot conclude that our figures represent the spontaneous course of untreated epilepsy because it is obvious that patients with mild epilepsy are more inclined to reject antiepileptic drug treatment than those with frequent seizures. The low likelihood of subsequent seizures in our patients may be partly explained by the relatively high proportion of patients with tonic-clonic seizures and cryptogenic epilepsy, factors which usually predict a good prognosis.

The proportion of untreated epileptic patients (4\%) in our study was smaller than previously reported from Poland $(36 \%)^{2}$ and United States (14\%). Our case ascertainment methods were probably less sensitive in identifying patients not seeking medical treatment for their seizures than were the household survey methods applied in previous studies. ${ }^{24}$ Even so, all people with epilepsy identified in an Australian household study had been prescribed antiepileptic drugs." Thus, there may be true variation in the prevalence of untreated epilepsy in different countries.

The predominance of men among the untreated patients was more pronounced than in the total epileptic population.' The finding may reflect different patterns of seeking medical help in men and women.

1 Gowere WR. Bpilopsy and other convulsive disonders: their causes, symptoms, and treatment. London, Churchill, 1881.

2 Zielinaki J. Epileptics not in treament. Bpilopsia 1974;15:203-10.

3 Kertnen T, Riekkinen PJ, Sillanpta M. Incidence and prevalence of epilepay in adults in Eastern Finland. Epilepsia 1989;30:413-21.

4 Haerer AF, Anderson DW, Schoenberg BS. Prevalence and clinical features of epilepay in a biracial United States population. Epilopsia 1986;27:66-75.

5 Beran RG, Hall L, Pesch A, Ho P, Lam S, Leung P-Y, at al. Population prevalence of epilepay in Sydney, Australia. Neuroopidemiology 1982;1:201-8.

(Accopted 11 May 1993) 